\title{
The owl's cochlear nuclei process different sound localization cues
}

\author{
Masakazu Konishi, W. Edward Sullivan, and Terry Takahashi \\ Division of Biology 216-76, California Institute of Technology, Pasadena, California 91125
}

(Received 1 January 1984; accepted for publication 22 February 1985)

This paper discusses how the barn owl's brain stem auditory pathway is divided into two physiologically and anatomically segregated channels for separate processing of interaural phase and intensity cues for sound localization. The paper also points out the power of the "downstream" approach by which the emergence of a higher-order neuron's stimulus selectivity can be traced through lower-order stations.

PACS numbers: 43.10.Ln, 43.63.Qe, 43.63.Th, 43.63.Bq

\section{INTRODUCTION}

Why is there more than one cochlear nucleus? By itself, characterization of differences in the physiology and anatomy of different nuclei cannot answer the question. These differences must be understood in the context of behavior. For example, without understanding which variables of the acoustic stimulus (e.g., waveform timing, intensity, frequency or amplitude modulation, spectral patterns, and so on) carry information relevant to a specific behavior or perception, the search for neural codes has no logical focus. One must know what the auditory system has to encode before one can ask how it is coded. Also, for a given acoustic variable, there may be more than one possible neural code that could be used, and often there is no logical way to determine which one actually is used.

We advocate a "downstream" approach—carrying our analyses from higher-order, central neurons back down to the periphery. The higher-order neuron, our starting point, must satisfy the following four conditions: (1) It is highly selective for a complex, yet definable stimulus. (2) A definition of the stimulus variables to which the neuron is and is not sensitive can be achieved. (3) The animal's behavioral response to these same stimulus variables can be measured. (4) Lower-order neurons in the pathway leading to the highorder neuron can be identified.

We have studied the neural substrates of sound localization in the barn owl, and the downstream approach has enabled us to trace the auditory system's codes for space from the inferior colliculus down to the cochlear nuclei.

\section{WHAT CUES ARE USED IN SOUND LOCALIZATION?}

The owl reacts to a rustling noise by turning its head, in a rapid saccadic movement, toward the source of the sound. Measurement of this behavior with a speaker in free-field conditions showed that the owl localizes noises very accurately in azimuth and elevation (Knudsen et al., 1979). This bicoordinate localization requires binaural cues. Dichotic stimuli delivered through earphones induce the same orienting response, and this revealed that the owl uses interaural time (phase) and intensity differences to localize sound, respectively, in azimuth and in elevation (Moiseff and Konishi, 1981 and unpublished observations).
The time differences used by the owl range from a maximum of about $170 \mu \mathrm{s}$ to a minimum of about $10 \mu \mathrm{s}$. The owl's ability to use interaural intensity differences for elevation is due to the differential sensitivity of its ears. That is, for the frequency range (6-8 $\mathrm{kHz}$ ) best suited for sound localization the right and left ears are biased for sounds coming from, respectively, above and below the visual axis.

Thus the owl can assign a location in its auditory space to each combination of interaural time and intensity difference. In other words, these binaural cues define the owl's auditory space.

\section{THE SPACE-SPECIFIC CELL: A HIGHER-ORDER, COMPLEX NEURON}

A search for the neural basis of the barn owl's sound localization behavior led to the discovery of the "space-specific neuron," a neuron of the inferior colliculus which responds selectively to sounds emanating from a single, small area in space (Knudsen and Konishi, 1978a,b; see Knudsen, 1983 for the terminology of the owl's auditory midbrain). The spatial area from which a sound can excite a space-specific neuron is called the receptive field-an analogy to receptive fields in other sensory systems.

The mechanisms underlying auditory receptive fields, however, are different from those of receptive fields in other sensory systems (Konishi and Knudsen, 1982). Auditory receptive fields must be generated by assigning values of interaural disparities to actual field locations, rather than by the simple preservation of the receptor epithelium's surface geometry. As one might expect, space-specific neurons are also selective for a combination of interaural intensity difference and interaural timing difference, the cues the barn owl uses for sound localization. As illustrated in Fig. 1, a sound broadcast from the azimuthal center of the receptive field produces the interaural timing difference to which the spacespecific neuron is most responsive. Likewise, a sound originating from the elevational center of the receptive field produces the optimal interaural intensity difference. The size of the receptive field is determined by the width of the tuning curves, which can be quite narrow. Some show a 50\% decrease in response when either interaural time or intensity differences is changed, respectively, by $15 \mu \mathrm{s}$ or $5 \mathrm{~dB}$ (Moi- 

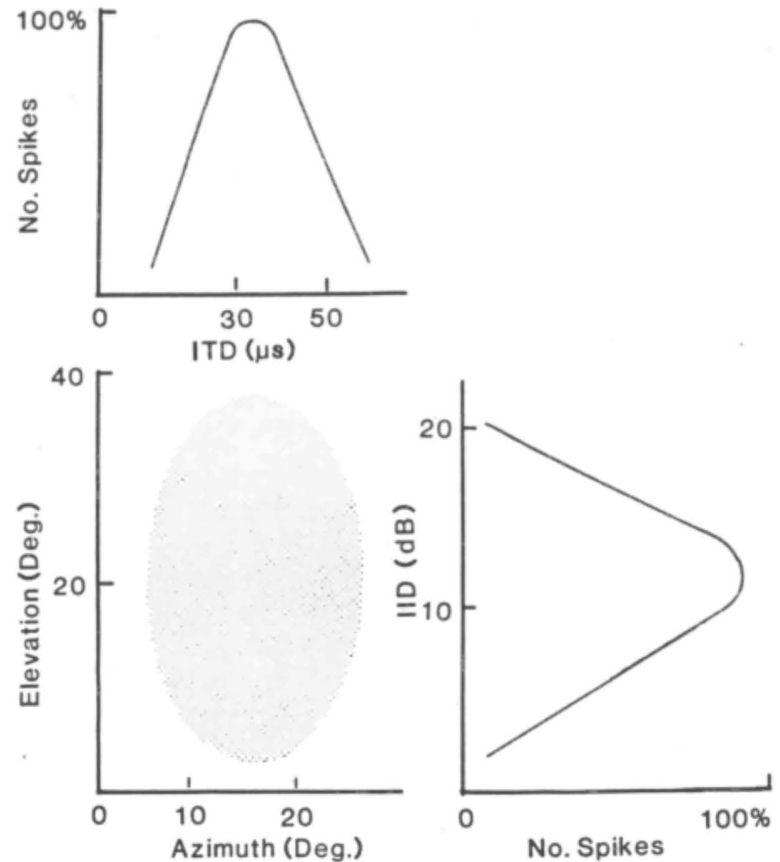

FIG. 1. Binaural cues for auditory receptive fields. A space-specific neuron requires a binaural stimulus containing a particular combination of interaural time (ITD) and intensity (IID) differences. This diagram is a scale model of a neuron's receptive field (shaded area) and its ITD and IID tuning curves.

seff and Konishi, 1981, 1984)—sharper than the tuning of any auditory neuron described in mammals (Rose et al., 1966; Kuwada and Yin, 1983).

\section{PATHWAYS TO THE SPACE-SPECIFIC CELL: NONOVERLAPPING TIME AND INTENSITY CHANNELS}

Having found the cues for sound localization and a class of neuron which is highly selective for these cues, we studied where the sensitivity to these cues originates in the lower auditory centers. An extensive survey of all binaural nuclei in the owl's brain stem revealed that no nucleus below the inferior colliculus contains neurons sensitive to both time and intensity disparities. Instead, binaural nuclei fit one of two categories; one, in which neurons are sensitive to interaural time differences, and the other, in which neurons are sensitive to interaural intensity differences (Moiseff and Konishi, 1984).

Nuclei sensitive to a particular interaural cue, moreover, appear to be interconnected, suggesting that there is a distinct neural pathway for each sound localization cue. Neuronal tuning to interaural time differences is first observed in $n$. laminaris, which is thought to be analogous to the mammalian medial superior olivary nucleus (MSO). $N$. laminaris receives bilateral inputs exclusively from one of the cochlear nuclei, n. magnocellularis, and it projects to one of the lemniscal nuclei, VLVa, where neurons are also tuned to interaural time differences, and to the inferior colliculus (Takahashi and Konishi, in preparation). Neuronal sensitivity (not tuning) to interaural intensity differences can be traced to a second nucleus of the lateral lemniscus, VLVp, which receives inputs from the contralateral n. angularis, the other cochlear nucleus (Takahashi and Konishi, in prepara- tion). Both VLVp and $n$. angularis project to the inferior colliculus.

The separation of time and intensity channels is also apparent in the response of the space-specific neuron. A space-specific neuron's selectivity for one interaural cue remains the same for all effective values of the other cue, indicating that the processing of one cue does not interfere with that of the other cue (Fig. 2). It is also possible to alter the selectivity of a space-specific neuron for one cue without affecting its selectivity to the other cue. While monitoring the selectivity of a space-specific neuron for the two interaural cues, one of the cochlear nuclei was anesthetized by an injection of lidocaine, a local anesthetic. Injection of $200 \mathrm{nl}$ of lidocaine into the cochlear nucleus blocks neural activity over a radius of approximately $300 \mu$ for a period of $15 \mathrm{~min}$, during which the anesthesia gradually wears off. Upon injection of lidocaine in the magnocellular nucleus, the spacespecific neuron becomes more broadly tuned to a new interaural time disparity, then gradually recovers. Meanwhile, there is no change in the neuron's tuning to interaural intensity disparity [Fig. 3(a)]. Anesthetizing the angular nucleus has the converse effect: the space-specific neuron's intensity disparity tuning curve is shifted and broadened while its tuning to interaural time disparity remains unchanged [Fig. 3(b)] (Takahashi et al., 1984). These findings indicate that time and intensity cues are processed in separate pathways which originate, respectively, in the magnocellular and angular nuclei.

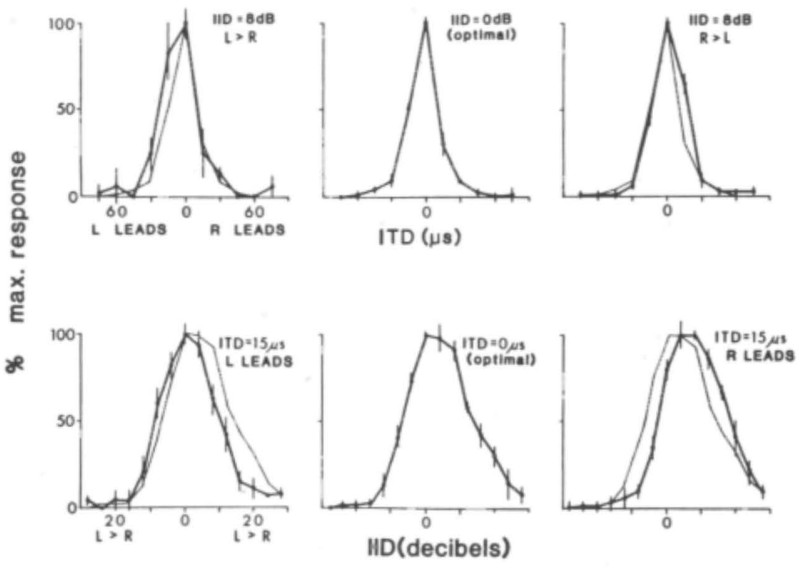

FIG. 2. Independence of time and intensity processing. The selectivity of a space-specific neuron for one interaural cue is not influenced by the value of the other cue, as long as the other cue is effective. The average number of spikes per stimulus (noise) presentation (five repetitions), normalized to the maximum response, is plotted against interaural intensity difference (IID) or interaural time difference (ITD) in each graph of this figure and of Fig. $3(a)$ and (b). The vertical bars represent the standard deviation. The top row of this figure shows the results of an experiment in which the unit's selectivity to ITD was monitored while the IID was set at the optimal value (center graph) or at nonoptimal values (adjacent graphs). The bottom row depicts the results of the converse experiment in which the unit's selectivity for IID was monitored while the ITD was set at the optimal value (center graph) or at nonoptimal values (adjacent graphs). The curves obtained under optimal conditions are superimposed on adjacent graphs to facilitate comparison. In both cases, the curves obtained under nonoptimal conditions are no different from those obtained under optimal conditions. 

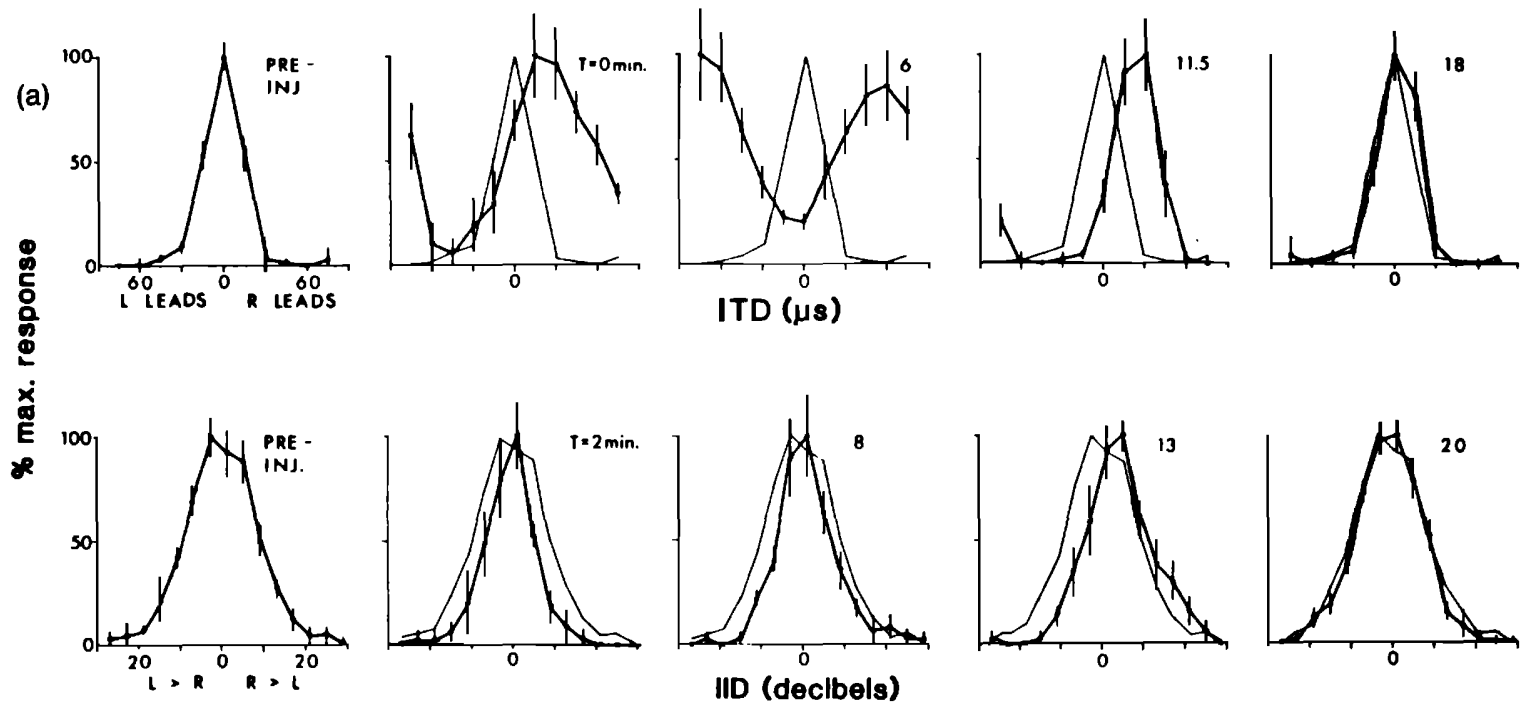

ITD ( $\mu s)$
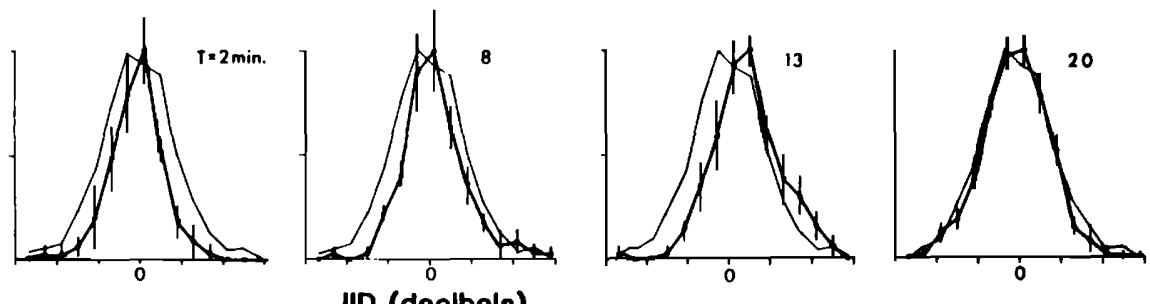

IID (declbels)
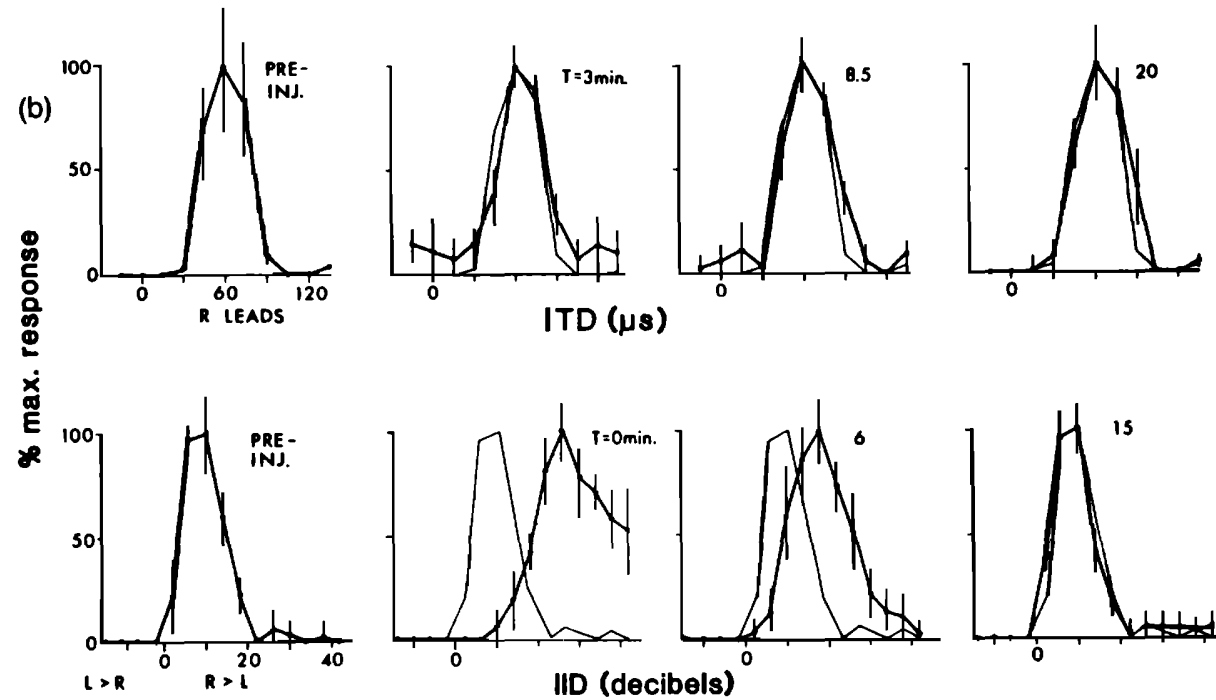

FIG. 3. (a) Injection of lidocaine, a local anesthetic, into nucleus magnocellularis alters a space-specific unit's selectivity for ITD but not its selectivity for IID. Before lidocaine injection (Pre-inj.), the cell is selective for noise stimuli of equal intensities presented simultaneously to the ears (IID $=0 \mathrm{~dB}$; ITD $=0 \mu$ s). Upon injection $(T=0 \mathrm{~min})$, the cell becomes responsive to two, different ITD values $(T=0 \mathrm{~min}$ to $T=6 \mathrm{~min})$, then recovers over the next $12 \mathrm{~min}$ ( $T=6 \mathrm{~min}$ to $T=18 \mathrm{~min}$ ). Its selectivity for IID remains unchanged throughout. The graph of the unit's response to ITD or IID obtained prior to lidocaine injection is superimposed on each of the other graphs for easy comparison in this figure and in part (b). (b) Injection of lidocaine into nucleus angularis alters a spacespecific neuron's selectivity for interaural intensity difference (IID), but not its selectivity for interaural time difference (ITD). Prior to lidocaine injection, the space-specific unit responds best when the noise stimulus in the right ear leads by $60 \mu \mathrm{s}$ and is $12 \mathrm{~dB}$ louder. The unit, moreover, is highly selective for these values-its response declines to $50 \%$ of the maximum if the ITD or IID is changed by $15 \mu \mathrm{s}$ or $6 \mathrm{~dB}$, respectively. Upon injection ( $T=0$ min), the spacespecific neuron becomes less selective and responds best when the right ear is $22 \mathrm{~dB}$ louder. It regains its sharp selectivity to the original IID in the ensuing 15 $\min (T=0$ to $T=15 \mathrm{~min})$. The unit's selectivity for ITD, meanwhile, is unchanged.

\section{THE CODES FOR TIME AND INTENSITY IN THE COCHLEAR NUCLEI}

Both the owl and its space-specific neurons are sensitive to microsecond interaural time differences of high-frequency $(6-9 \mathrm{kHz})$ sounds. This suggests that the owl is able to encode the temporal pattern of the signal waveform with microsecond accuracy. A search for neurons capable of such coding focused upon the magnocellular nucleus because it innervates nucleus laminaris, the lowest brain stem structure displaying tuning to interaural time disparities. This study showed that virtually all neurons in the owl's magnocellular nucleus fire at a particular phase of a tonal stimulus. This "phase locking" occurs not only in the low-frequency range as in mammals, but also in the high-frequency range which the owl uses for sound localization. The degree of phase locking decreases as a unit's characteristic frequency increases [Fig. 4(a)] and disappears at $9 \mathrm{kHz}$, a finding consistent with the fact that the owl cannot accurately localize tones of frequencies higher than about $9 \mathrm{kHz}$. Binaural neurons tuned to this frequency range also fail to show sensitivity to interaural time differences. In sharp contrast to magnocellular neurons, the overwhelming majority of cells in the angular nucleus show no phase locking. Those that do, have low best frequencies, and the degree of their phase locking is far below those of low-frequency, magnocellular cells [Fig. 4(b)]. 


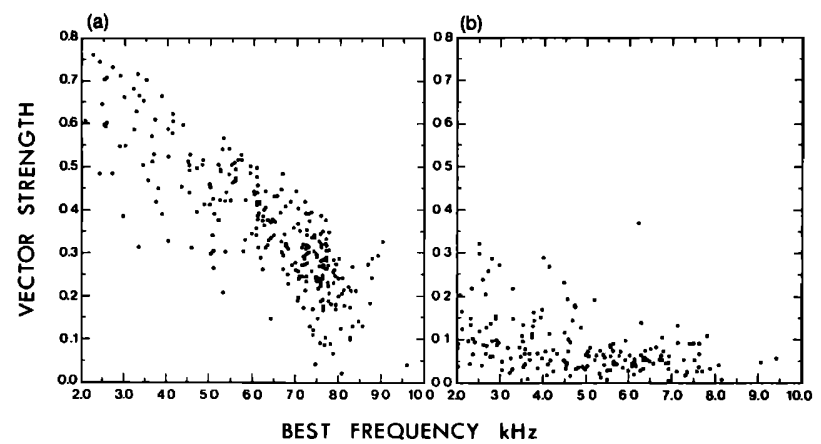

FIG. 4. Phase locking or vector strength plotted as a function of unit best frequency for 296 magnocellular neurons (a), and 179 angular neurons (b). Vector strength was calculated according to the method of Goldberg and Brown (1969). The value chosen for a given unit was the value for the response at best frequency and $20 \mathrm{~dB}$ above threshold. A value of 1.0 indicates perfect phase locking and 0.0 indicates random discharge with reference to stimulus phase. The vector strengths of magnocellular neurons are higher than those of angular neurons at frequencies up to about $9 \mathrm{kHz}$. The vector strength values for angular neurons are usually less than 0.1 and these values can be explained by random spike distributions rather than by low levels of phase locking.

Thus, neurons in the magnocellular nucleus are well suited to provide information on waveform timing in the timing of their discharges, whereas those of the angular nucleus are not (Sullivan and Konishi, 1984).

By contrast, the cells of nucleus angularis appear to be well adapted for encoding stimulus intensity. The spike rate of these neurons increases rapidly and reliably as sound intensity increases over a wide range $(25-40 \mathrm{~dB})$. The discharge patterns of these neurons are very regular, implying that the relative timing of one spike to the next is a stable feature of their response. This would mean that higher centers can extract reliable information on spike rate in a short time period. As shown in Fig. 5, the neurons of nucleus magnocellularis show changes in response over smaller ranges of intensity with less sensitivity and more response variability than do those of nucleus angularis.

Two other characteristics of a spike train, response latency and absolute spike number, are also functions of sound intensity and have been suggested as putative codes. Response latency, however, is also dependent upon the arrival time of sounds and absolute spike number is also affected by stimulus duration. Neither changes in the relative arrival time of the sound at each ear nor changes in the duration of sounds affect the owl's ability to localize in elevation (Knudsen and Konishi, 1979; and Moiseff and Konishi, 1981).

\section{COMPARISON WITH OTHER AUDITORY SYSTEMS}

There are differences as well as similarities between the barn owl's auditory system and other vertebrate auditory systems. In the owl, phase locking is observed at frequencies about an octave higher than the maximum frequency for which phase locking can be observed in mammalian neurons (Rose et al., 1967). In mammals, it is assumed that the localization of low frequencies is achieved by interaural time comparison whereas high frequencies are localized by the analysis of interaural differences in intensity. In the owl, however, the distinction between phase sensitive neurons
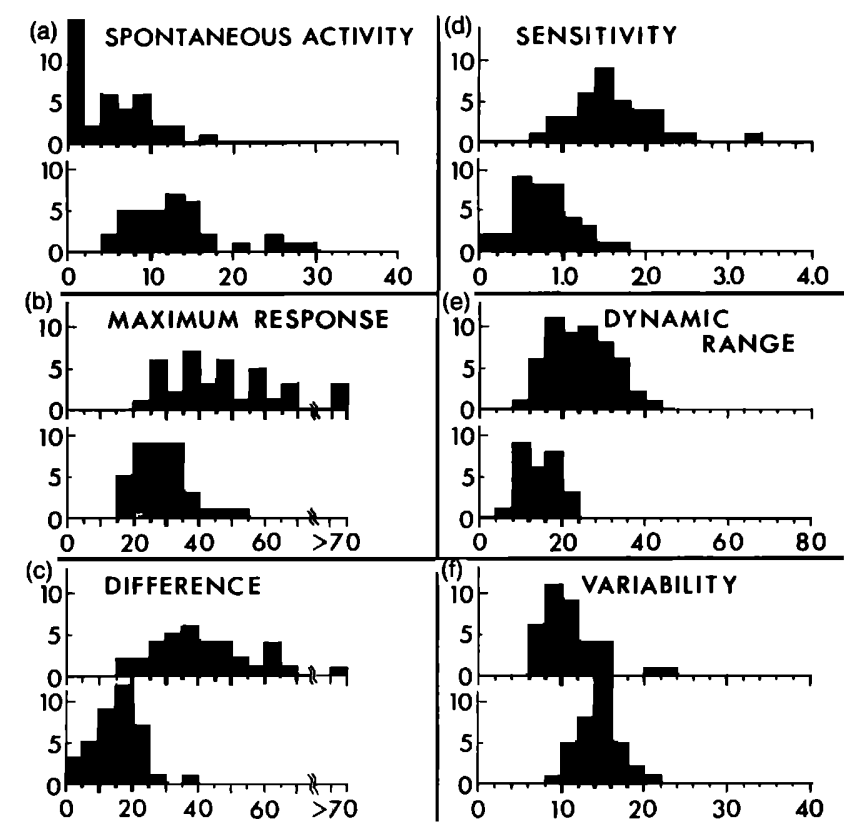

FIG. 5. Population comparison of neuronal sensitivity to stimulus intensity between $n$. angularis (the top histogram in each pair) and n. magnocellularis (bottom). (a) Average spontaneous activity in spikes $/ 150 \mathrm{~ms}$. Mean value for $n$. angularis (n.a.) population $=6.21 \pm 4.36$; mean value for $n$. magnocellularis (n.m.) population $=14.16 \pm 5.85$. (b) Maximum response level for a 100 -ms tone burst in spikes $/ 150$ ms. Mean for n.a. $=48.68 \pm 14.96$; mean for n.m. $=32.63 \pm 11.19$. (c) Difference between maximum response and spontaneous level (spikes $/ 150 \mathrm{~ms}$ ). Mean for n.a. $=44.03 \pm 14.68$; mean for n.m. $=18.03 \pm 7.22$. (d) Average sensitivity in spike counts/dB for change in response within the unit's dynamic range. Mean for n.a. $=1.64 \pm 0.50$; mean for n.m. $=0.82 \pm 0.36$. (e) Dynamic range in dB estimated to the nearest $4 \mathrm{~dB}$. Mean for n.a. $=26.30 \pm 7.36$; mean for n.m. $=16.44 \pm 4.48$. (f) Response variability: the ratio of spike count standard deviation to average spike counts in percent, at a response level of 20 30 spikes/stimulus. Mean for n.a. $=12.00 \pm 3.64$; mean for n.m. $=15.50 \pm 2.55$.

and intensity sensitive neurons is not made on the basis of frequency tuning. Neuronal responses also seem to be more homogeneous in the owl's cochlear nuclei than in the mammalian cochlear nuclei which display a variety of responses (Kiang et al., 1973; Sullivan and Konishi, 1984).

There are also some similarities between some of the subdivisions of the mammalian cochlear nucleus and the owl's cochlear nuclei. The magnocellular nucleus and the anterior division of the anteroventral cochlear nucleus in mammals both contain "bushy" cells which receive calycine terminals from auditory nerve fibers and show phase locking (Brawer et al., 1974; Jhaveri and Morest, 1982; Goldberg and Brownell, 1974; Sullivan and Konishi, 1984). Neurons of nucleus angularis and the stellate cell population in the mammalian ventral cochlear nucleus are similar in that both show little phase locking and display "chopper" type poststimulus time (PST) histograms (Pfeiffer, 1966; Rhode et al., 1983; Sullivan, 1985). In both angular neurons and stellate neurons, auditory nerve fibers terminate as small boutonal endings presumably upon both dendrites and somata (Molnar and Pfeiffer, 1968; Brawer and Morest, 1975; Takahashi, unpublished observations).

These morphological and physiological similarities between the owl's cochlear nuclei and divisions of the mam- 
malian ventral cochlear nucleus suggest that they function in similar capacities. However, more comparative behavioral data are required to substantiate this hypothesis. The barn owl's natural head turning behavior provided us with a convenient method of studying the acoustic variables involved in its sound localization. Until a comparable level of behavioral analysis is achieved for the cat or monkey, we can only speculate on the functions of their brain stem auditory nuclei in sound localization.

\section{ACKNOWLEDGMENTS}

This work was supported by NIH grant NS 14617 to M.K., NIH postdoctoral fellowship NS 07045-01 to W. E. S., and a Del Webb postdoctoral fellowship to T. T.

Brawer, J. R., Morest, D. K., and Kane, E. C. (1974). "The neuronal architecture of the cochlear nucleus of the cat," J. Comp. Neurol. 155, 251300.

Brawer, J. R., and Morest, D. K. (1975). "Relations between auditory nerve endings and cell types in the cat's anteroventral cochlear nucleus seen with the Golgi method and Nomarski optics," J. Comp. Neurol. 160, 491-506.

Goldberg, J. M., and Brown, P. B. (1969). "Responses of binaural neurons of dog superior olivary complex to dichotic tonal stimuli: Some physiological mechanisms of sound localization," J. Neurophysiol. 32, 613-636.

Goldberg, J. M., and Brownell, W. E. (1974). "Discharge characteristics of neurons in anteroventral and dorsal cochlear nuclei of cat," Brain Res. 64, 35-54.

Jhaveri, S., and Morest, D. K. (1982). "Neuronal architecture in nucleus magnocellularis of the chicken auditory system with observations on nucleus laminaris: A light and electron microscope study," Neurosci. 7, 809-836.

Kiang, N. Y. S., Morest, D. K., Godfrey, D. A., Guinan, J. J., and Kane, E. C. (1973). "Stimulus coding at caudal levels of the cat's auditory nervous system. I. Response characteristics of single units," in Basic Mechanisms in Hearing, edited by A. R. Møller (Academic, New York), pp. 455-478.

Knudsen, E. I. (1983). "Subdivisions of the inferior colliculus in the barn owl (Tyto alba)," J. Comp. Neurol. 218, 174-186.

Knudsen, E. I., Blasdel, G. G., and Konishi, M. (1979). "Sound localization by the barn owl (Tyto alba) measured with the search coil technique," J. Comp. Physiol. 133, 1-11.

Knudsen, E. I., and Konishi, M. (1978a). "Space and frequency are represented separately in auditory midbrain of the owl," J. Neurophysiol. 41, $870-884$.

Knudsen, E. I., and Konishi, M. (1978b). “A neural map of auditory space in the owl," Science 200, 795-797.

Knudsen, E. I., and Konishi, M. (1979). "Mechanisms of sound localization in the barn owl (Tyto alba)," J. Comp. Physiol. 133, 13-21.

Knudsen, E. I., and Konishi, M. (1980). "Monaural occlusion shifts receptive-field locations of auditory midbrain units in the owl," J. Neurophysiol. 44, 687-695.

Konishi, M., and Knudsen, E. I. (1982). “A theory of neural auditory space: Auditory representation in the owl and its significance," in Cortical Sensory Organization, Vol. 3, Multiple Auditory Areas," edited by C. N. Woolsey (Humana, Clifton, NJ).

Kuwada, S., and Yin, T. C. T. (1983). "Binaural interaction in low-frequency neurons in inferior colliculus of the cat. I. Effects of long interaural delays, intensity, and repetition rate on interaural delay function," J. Neurophysiol. 50, 981-999.

Moiseff, A., and Konishi, M. (1981). "Neuronal and behavioral sensitivity to binaural time differences in the owl," J. Neurosci. 1, 40-48.

Moiseff, A., and Konishi, M. (1984). "Binaural characteristics of units in the owl's brainstem auditory pathways: Precursors of restricted spatial receptive fields," J. Neurosci. (in press).

Molnar, C. E., and Pfeiffer, R. R. (1968). "Interpretation of spontaneous spike discharge patterns of neurons in the cochlear nucleus," Proc. IEEE 56, 993-1004.

Pfeiffer, R. R. (1966). "Classification of response patterns of spike discharges for units in the cochlear nucleus: Tone-burst stimulation," Exp. Brain Res. 200, 220-235.

Rhode, W. S., Oertel, D., and Smith, P. H. (1983). "Physiological response properties of cells labeled intracellularly with horseradish peroxidase in cat ventral cochlear nucleus,"' J. Comp. Neurol. 213, 448-463.

Rose, J. E., Gross, N. B., Geisler, C. D., and Hind, J. E. (1966). "Some neural mechanisms in the inferior colliculus of the cat which may be relevant to localization of a sound source," J. Neurophysiol. 29, 288-314.

Sullivan, W. E. (1985). "Classification of response patterns in cochlear nucleus of barn owl: Correlation with functional response properties," J. Neurophysiol. 53, 201-216.

Sullivan, W. E., and Konishi, M. (1984). "Segregation of stimulus phase and intensity coding in the cochlear nucleus of the barn owl," J. Neurosci. 4, 1787-1799.

Takahashi, T., Moiseff, A., and Konishi, M. (1984). "Time and intensity cues are processed independently in the auditory system of the owl," $J$. Neurosci. 4, 1781-1786. 